\title{
All Optical Generation and Processing of IR UWB Signals
}

\author{
Y. Ben Ezra, B.I. Lembrikov, M. Ran and M. Haridim \\ Holon Institute of Technology (HIT), \\ P.O.Box 305, 58102, 52 Golomb Str., Holon \\ Israel
}

\section{Introduction}

\subsection{Impulse Radio (IR) Ultra Wideband (UWB) Communications}

Ultra wideband (UWB) communication is a fast emerging technology that offers new opportunities such as high data rates, low equipment cost, low power, precise positioning capability and extremely low interference Ghawami (2005). A wide range of possible UWB communication system applications includes radars due to UWB ultra high precision ranging at the centimeter level, wireless personal area networks (WPAN), sensor networks, imaging systems, UWB positioning systems, etc. Yang (2004), Kshetrimayum (2009). From a commercial point of view, the high data rates are the most attractive feature of UWB systems for which speeds of over $100 \mathrm{Mb} / \mathrm{s}$ have been demonstrated Ghawami (2005). There exist three main types of UWB technologies: impulse radio (IR UWB), direct sequence (DS UWB), and multi-band orthogonal frequency division multiplexing (MB OFDM) Ran (2009). IR UWB communication technique is essentially different from all other communication techniques because it is carrier free and uses for communication between transmitters and receivers very narrow radio frequency (RF) pulses generated from the UWB pulse generator, while traditional transmission systems transmit information by varying the power, frequency, and/or phase of a sinusoidal wave in a modulation process Kshetrimayum (2009), Yao (2007). Consequently, complicated frequency mixers and local oscillators for carrier frequency up and down conversion are not necessary Yao (2007). IR UWB modulation techniques are especially important because the UWB spectrum has been made available by the Federal Communication Commission (FCC) and it can be used with IRs developed to date Lin (2005).

Waveforms for IR UWB are designed to obtain a flat frequency response over the bandwidth of the pulse and to avoid a DC component. The large spectrum of a UWB signal may interfere with existing users. In order to keep this interference to the minimum, FCC specifies spectral masks for different applications, i.e. the allowed power output for specific frequencies Ghawami (2005). A contiguous bandwidth of $7.5 \mathrm{GHz}$ is available in the frequency interval of $(3.1-10.6) \mathrm{GHz}$ at a maximum power output of $-41.3 \mathrm{dBm} / \mathrm{MHz}$ which is considered as extremely low Ghawami (2005). The power spectral density (PSD) of UWB systems defined as a ratio of the transmitted power $P$ in watts and a signal bandwidth $B$ in hertz is extremely low as compared to other communication systems due to the very wide bandwidth $B$ of short pulses that are typically of nanosecond or picosecond order Ghawami (2005). The selection of the impulse signal type for IR UWB communication system is essential since it determines 
the system performance Yao (2007). The fast switching in UWB systems results in a nonrectangular pulse shape which can approximated by a zero-mean Gauss function $G(x)$ with a standard deviation $\sigma$ Ghawami (2005)

$$
G(x)=\frac{1}{\sqrt{2 \pi \sigma^{2}}} \exp \left(-\frac{x^{2}}{2 \sigma^{2}}\right)
$$

A whole class of waveforms are called Gaussian waveforms due to their similarity to the Gauss function (1). The basis of these Gaussian waveforms is a Gaussian pulse given by Ghawami (2005)

$$
y_{g 1}=K_{1} \exp \left(-\frac{t^{2}}{\tau^{2}}\right)
$$

where $-\infty<t<\infty, \tau$ is the time-scaling factor, and $K_{1}$ is a constant. A Gaussian monocycle $y_{g 2}$ and a Gaussian doublet $y_{g 3}$ are given by the first and the second derivative of a Gaussian pulse, respectively Ghawami (2005)

$$
y_{g 2}=K_{2}\left(-\frac{2 t}{\tau^{2}}\right) \exp \left(-\frac{t^{2}}{\tau^{2}}\right) ; y_{g 3}=K_{3}\left(-\frac{2}{\tau^{2}}\right)\left(1-\frac{2 t^{2}}{\tau^{2}}\right) \exp \left(-\frac{t^{2}}{\tau^{2}}\right)
$$

For the energies $E_{1,2,3}$ of the Gaussian pulses $y_{g 1}, y_{g 2}, y_{g 3}$, the normalization constants $K_{1,2,3}$ can be evaluated as follows.

$$
K_{1}=\sqrt{\frac{E_{1}}{\tau \sqrt{\pi / 2}}} ; K_{2}=\sqrt{\frac{\tau E_{2}}{\sqrt{\pi / 2}}} ; K_{3}=\tau \sqrt{\frac{\tau E_{3}}{3 \sqrt{\pi / 2}}}
$$

The spectra of the Gaussian pulses (2), (3) determined by their Fourier transforms $Y_{g 1}(f)$, $Y_{g 2}(f), Y_{g 3}(f)$, respectively, have the form Ghawami (2005)

$$
\begin{gathered}
Y_{g 1}(f)=K_{1} \tau \sqrt{\pi} \exp \left[-(\pi \tau f)^{2}\right] \\
Y_{g 2}(f)=K_{2} \tau \sqrt{\pi}(j 2 \pi f) \exp \left[-(\pi \tau f)^{2}\right] \\
Y_{g 3}(f)=-K_{3}(2 \pi f)^{2} \exp \left[-(\pi \tau f)^{2}\right]
\end{gathered}
$$

Gaussian pulses are the most widely used waveforms due to their simplicity and achievability Yao (2007). The important property of the Gaussian pulses (2), (3) is that they are almost uniformly distributed over their frequency spectrum Ghawami (2005). UWB waveform can be generated by passing a Gaussian pulse through a bandpass filter acting similar to a first- or second-order differentiator Ghawami (2005), Yao (2007). However, it is difficult and expensive to generate such pulses with a fractional bandwidth greater than $100 \%$ at the central frequency of about $7 \mathrm{GHz}$ Yao (2007).

The current high data rate UWB systems are inherently limited to short-ranges of less than $10 m$ due the constraints on allowed emission levels Yao (2007), Ran (2009). The transmission of high data rate UWB signals over larger distances of about $10-10000 \mathrm{~m}$ is needed for broadband access technology. In order to increase the IR UWB transmission distances, a new concept combining the high data rate wireless short-range communications based on UWB technologies and the optical fiber technology has been proposed Ran (2009). This concept is called UWB radio over optical fibre (UROOF) Ran (2009). In this approach, the IR UWB signals of several $\mathrm{GHz}$ are superimposed on the optical continuous wave (CW) carrier and 
transmitted transparently over an optical fiber Ran (2009), Yao (2007).The UROOF technology permits to avoid the high cost additional electronic components required for signal processing and makes it possible the integration of all the RF and optical transmitter/receiver components on a single chip. The overall combination of these features results in a cost-effective broadband system that can easily support $1 \mathrm{~Gb} / \mathrm{s}$ suitable for WPAN applications Ran (2009). Previously, most of the approaches to generating of IR UWB signals were based on electronic methods Wang (2006). UWB pulses can be generated electrically using electronic circuits such as a microwave ring filter, a microwave resonator-based bandpass filter, or a microwave differentiator Yao (2007). However, in order to distribute UWB signals over the optical fiber, it is desirable to generate these signals directly in the optical domain without extra electricalto-optical (E/O) conversion Yao (2007). Recently, optically based methods of the Gaussian IR UWB monocycles and doublets generation for low-cost high-data rate UWB wireless systems have been proposed Lin (2005), Le Guennec (2007), Yao (2007), Zeng (April 2006), Zeng (October 2006), Zeng (2007). The advantages of these methods are following: the decreasing of interference between electrical devices, low loss and light weight of optical fibers Lin (2005), Yao (2007), Wang (2006). Below, in section 1.2, we consider the existing techniques of all-optical IR UWB generation.

\subsection{Optical Techniques of IR UWB Generation}

Optical IR UWB generation techniques are divided into 3 groups Yao (2007)

1. UWB pulse generation based on phase-modulation-to-intensity-modulation (PM-IM) conversion.

2. UWB pulse generation based on a photonic microwave delay line where the cross gain modulation (XGM) in a semiconductor optical amplifier (SOA) is used.

3. UWB pulse generation based on optical spectral shaping and dispersion-induced frequency-to-time mapping.

All these techniques can be implemented using fiber optic components such as an electro-optic phase modulator (EOM), a singlemode fiber (SMF), Erbium doped fiber amplifier (EDFA), a fiber Bragg grating (FBG) and a photodetector (PD) which provide the potential for integration based on integrated photonic circuits Yao (2007), Wang (2006), Zeng (April 2006), Zeng (October 2006). It should be noted that PM-IM conversion techniques are simpler than the techniques related to groups 2 and 3 while the approach based on the group 3 techniques has more flexibility in generating UWB pulses with arbitrary shapes Yao (2007). Recently novel UWB pulse generation techniques have been developed where UWB pulses were directly generated in the optical domain and distributed over optical fiber without the need for an extra E/O conversion Yao (2007).

In this work, we concentrate on the all-optical methods of UWB pulse generation based on nonlinear optical processes in SOA such as cross phase modulation (XPM) and XGM. Consider first the method based on XPM. The experimental setup for all-optical UWB doublet generation is shown in Fig. 1 Dong (2009). A probe CW signal is generated by CW laser diode at the wavelength $\lambda=1550.56 \mathrm{~nm}$ with a $1 \mathrm{~mW}$ output power. A tunable laser emits CW light at $\lambda=1558.7 \mathrm{~nm}$ modulated by the Mach-Zehnder modulator (MZM) in order to form optical Gaussian pulses of the type (2). The bit pattern generator (BPG) drives the MZM at a repetition rate of $20 \mathrm{~Gb} / \mathrm{s}$ with a fixed pattern equivalent to a Gaussian pulse train $P_{S}(t)$ with a 
repetition rate of $1.25 \mathrm{GHz}$ given by Dong (2009)

$$
P_{S}(t)=\sum_{n=-\infty}^{\infty} \Omega\left(t-n T_{r}\right)
$$

where $\Omega(t)$ is an ideal Gaussian waveform of the type (2) with a duty cycle of about $1 / 16$ and full width at half maximum (FWHM) of about $50 \mathrm{ps}$, and $T_{r}$ is the pulse repetition interval. When the Gaussian pulses (8) and the probe signal are simultaneously fed into SOA, the probe signal will undergo both XGM and XPM, and the phase $\Phi_{c}$ is varying approximately proportionally to $P_{\mathcal{S}}(t)$ Dong (2009)

$$
\Phi_{c}=K P_{S}(t)+\Phi_{0}
$$

where $K$ is the proportionality constant and $\Phi_{0}$ is the initial phase. The chirp $\Delta v_{\mathcal{c}}(t)$ of the probe signal is the first order derivative of the phase given by Dong (2009)

$$
\Delta v_{c}(t)=-\frac{1}{2 \pi} \frac{d \Phi_{c}}{d t}=-\frac{K}{2 \pi} \frac{d P_{s}(t)}{d t}
$$

The chirp (10) is a monocycle, according to definition (3). Its positive or negative value corresponds to blue or red shift of the probe wavelength, respectively Dong (2009). The SOA is followed by Gaussian optical blue shifted optical bandpass filter (OBF) and red shifted OBF forming the two polarity-reversed monocycle pulses with an opposite detuning to the probe carrier $\lambda_{c}$. Then, UWB doublet pulses can be obtained by combining the positive and negative monocycles with a proper delay Dong (2009). EDFA is necessary to amplify the output optical signal power due to large loss of OBFs, and OBF at the output with $1 \mathrm{~nm}$ bandwidth is used in order to suppress the amplified spontaneous emission (ASE) noise Dong (2009).

The necessity of OBFs and EDFA makes the UWB pulse generation system based on XPM a costly one Dong (2009). Two polarity-reversed pulses can be obtained with a pump-probe scheme based on XGM in SOA where the time delay between the pump and probe waves is appropriately chosen. The experimental setup for the realization of such a scheme is shown in Fig. 2 Dong (2009). A CW probe light at $\lambda_{c}$ and an optical Gaussian pulse train (8) at the wavelength $\lambda_{s}$ are emitted by a CW laser diode and a tunable laser externally modulated by MZM, respectively, and launched into the SOA simultaneously. Two attenuators (ATTs) are used for the SOA input power adjustment. The CW probe signal obtains an inverted Gaussian shape due to XGM in the SOA. The peak of the Gaussian pulse and the pit of the probe signal at the SOA output are misaligned due to the non-uniform amplification which results in a monocycle shape without optical filter and time delay Dong (2009). A SMF section of $2.5 \mathrm{~km}$ serves as a dispersion medium in order to induce a group delay between the optical Gaussian pulse and the inverted probe light. Both positive and negative monocycle pulses can be obtained depending on the SMF group delay Dong (2009).

All-optical UWB pulse generation based on a hybrid of self phase modulation (SPM) and $X \mathrm{XM}$, has also been demonstrated where the monocycle pulse is generated from a dark returnto-zero (RZ) signal and converted to a doublet pulse by injecting an additional probe signal with the SMF transmission Dong (2009).

An alternative approach based on optical cross polarization modulation has been recently proposed and demonstrated Chen (2008). Optical signal is cross-polarized modulated with simple electric pulses. An electrical pulse is used to drive an optical phase modulator (PM) where the phase shift between the two optical axes depends on the drive voltage $V$. If the 


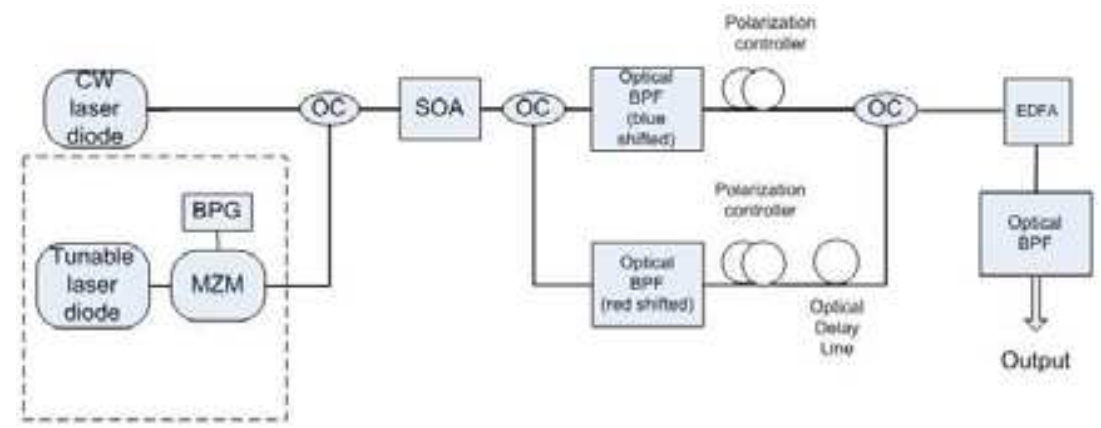

Fig. 1. Experimental setup for all-optical UWB generation based on XPM scheme (BPG: bit pattern generator; OC: optical coupler; MZM: Mach-Zehnder modulator; OBF: optical bandpass filter; ODL: optical delay line; PC: polarization controller)

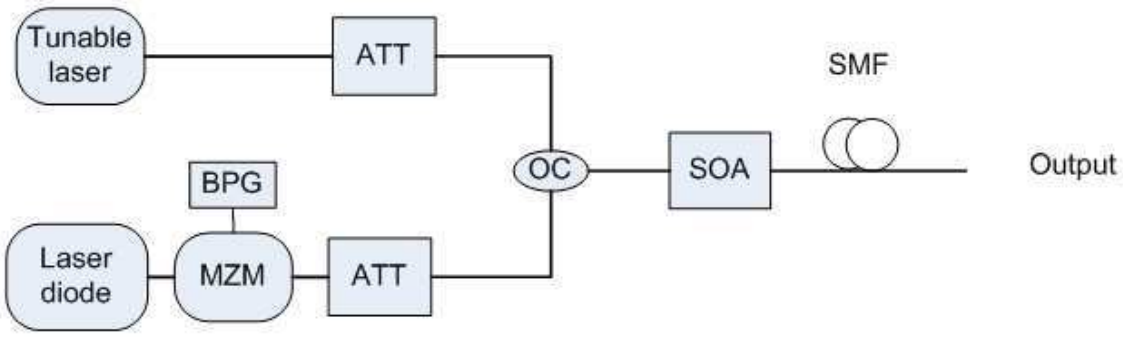

Fig. 2. Experimental setup for filter-free UWB generation based on XGM scheme (ATT: attenuator; SMF: single mode fiber; other abbreviations are similar to 1)

voltage $V=V_{\pi}$, then the polarization will rotate to the orthogonal direction at the output of PM. As a result, at the original input orientation there exists simultaneously a negative pulse. This process is called cross-polarization modulation (CPM) Chen (2008). Then, the monocycle pulses are obtained after a proper birefrigence time delay and optical-to-electrical $(\mathrm{O} / \mathrm{E})$ conversion, and doublet pulses are obtained by using gain saturation and recovery processes in SOA Chen (2008).

The shortages of the proposed methods are the necessity of the sophisticated circuit for the generation of short electric Gaussian pulses, the use of EOM, and the need for a comparatively long SMF. Bulk SOAs are characterized by a comparatively low operation rate and high bias currents.

We proposed a theoretical analysis of a novel all-optical method of the IR UWB pulse generation in an integrated Mach-Zehnder interferometer (MZI) with quantum dot semiconductor optical amplifier (QD SOA) as an active element inserted into one arm of the integrated MZI which results in an intensity dependent optical signal interference at the output of MZI Ben Ezra (2008). The IR UWB pulse generation process is based both on XPM and on XGM in QD SOA characterized by an extremely high optical nonlinearity, low bias currents, and high operation rate Sugawara (2004). Unlike other proposed all-optical methods, we need not optical fibers, FBG and EOM substantially reducing the cost and complexity of a IR UWB generator. The IR UWB signals generated by the proposed QD SOA based MZI structure have the 
form of the Gaussian doublet. The shape of the signal and its spectrum can be tailor-made for different applications by changing the QD SOA bias current and optical power.

Another novel approach of all-optical generation of UWB Gaussian monocycles and doublets is based on the system of two integrated unbalanced Mach-Zehnder Interferometers (MZIs) connected in parallel that does not contain any active elements. The MZIs are chosen in such a way that the phase difference of the interfering signals at the output is equal to $\pi$. Each one of the MZI is characterized by time delay differences $\tau$ and $2 \tau$. As a result, at the output of the MZIs the interfering optical signals modulated by a Gaussian UWB pulse form the first-order difference approximating the Gaussian monocycle and the second-order difference approximating the Gaussian doublet. At the output of the system the UWB monocycles and doublets can be converted to a UWB signal by means of a homodyne detection where the local oscillator frequency $\omega_{L O}$ coincides with the optical carrier frequency $\omega$ Agrawal (2002). In such a case the homodyne detected signal is proportional to the UWB modulated optical signal amplitude Agrawal (2002), i.e. to the Gaussian monocycle or doublet in our case. The analysis shows that the performance of the proposed system is stable with respect to reasonable deviations of the MZI arm length and an input coupler ratio. The system can be made tunable by including into MZIs a tunable delay line.

We also discuss an all-optical signal processing based on wavelet transform (WT) applications. We proposed the implementation of the WT analysis for all-optical signal denoising, and alloptical passive generation of a so-called Mexican hat mother wavelet (MHMW) Rao (1998). The chapter is constructed as follows. The original results are presented in sections 2-6. The analysis of all-optical method of the IR UWB pulse generation in an integrated MZI with QD SOA can be divided into three stages. First, in section 2, we discuss the operation principle of unbalanced MZI (UMZI) containing QD SOA as a basic nonlinear component for the proposed scheme of all-optical IR UWB generation. Then, in section 3, ultra-fast nonlinear phenomena in QD SOA and are reviewed. Finally, in section 4, we present the simulation results for the IR UWB pulse generation in an integrated MZI with QD SOA. In sections 5 and 6, we propose a theory of all-optical passive generation of UWB Gaussian monocycles and doublets, all-optical passive generation of MHMW and all-optical signal denoising. The conclusions are presented in section 7.

\section{Mach-Zehnder Interferometer (MZI) Containing Nonlinear Elements}

There exist both all-fiber MZI Agrawal (2001) and monolithic integrated ones Joergensen (1996). The couplers at the input and output of MZI can have different power-splitting fractions, and two arms of MZI can have different lengths and propagation constants. Typically, in the linear case an input optical signal splits into two parts which acquire different phase if arm lengths are different. In such a case, MZI can be unbalanced since the two optical fields inside MZI have physically separated paths Agrawal (2001). Nonlinear applications of MZIs make use of the SPM- or XPM-induced phase shifts. The nonlinear response of an MZI can be switched from low to high or vice versa by changing the input peak power of the incident signal Agrawal (2001). The successful operation of integrated SOA-MZI structures in an essentially nonlinear regime providing wavelength conversion (WC), all-optical logic gate, and all-optical regeneration due to XGM has been demonstrated experimentally Chen (2002), Joergensen (1996), Kanellos (2007).

We discuss now the operation principle of the MZI containing in one arm a QD SOA as a nonlinear element. The block diagram of the proposed all-optical IR UWB pulse generator 
consisting of CW laser, a pulsed laser, and MZI with a QD SOA in its upper arm is shown in Fig. 3 Ben Ezra (2008).

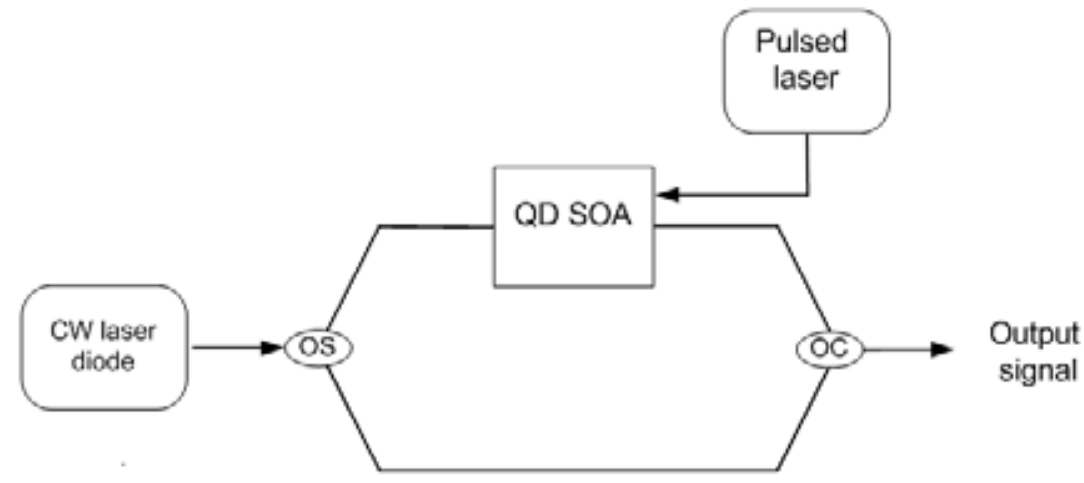

Fig. 3. Block diagram of the proposed IR UWB generator

A CW signal of a wavelength $\lambda$ and an optical power $P_{0}$ is split by an optical splitter (OS) into two signals with equal optical power and fed into the two ports of the integrated MZI one of which contains a QD SOA. In QD SOA XGM gain and XPM phase shift are related due to the linewidth enhancement factor (LEF) $\alpha$ Agrawal (1989). The pulsed laser produces a train of short Gaussian pulses counter-propagating with respect to the input CW optical signal. The CW signal propagating through the upper arm of MZI transforms into the Gaussian pulse at the output of the MZI due to XPM and XGM with the train of Gaussian pulses whereas the optical signal in the linear lower arm of MZI remains CW. Both these pulses interfere at the output of MZI, and the output pulse shape is defined by the power dependent phase difference $\Delta \phi(t)=\phi_{1}(t)-\phi_{2}(t)$ where $\phi_{1,2}(t)$ are the phase shifts in the upper and lower arms of MZI, respectively. Evidently, the phase shift in lower arm of MZI is constant: $\phi_{2}=$ const. The MZI output optical power $P_{\text {out }}$ is given by Wang (2004).

$$
P_{\text {out }}=\frac{P_{0}}{4}\left[G_{1}(t)+G_{2}(t)-2 \sqrt{G_{1}(t) G_{2}(t)} \cos \Delta \phi(t)\right]
$$

where $G_{1,2}(t)$ are amplification factors of the upper and lower arms of MZI. The upper arm amplification factor $\exp \left(g_{\text {sat }} L\right) \leq G_{1}(t)=\exp (g L) \leq \exp \left(g_{0} L\right)$ defined by the QD SOA gain $g$ and its active region length $L$ is limited by the saturation gain $g_{\text {sat }}$ and the maximum modal gain $g_{0}$ typical for the linear regime. The linear lower arm amplification factor is simply unity $G_{2}=1$. The losses can be neglected due to a small length of integrated elements. Then the relation between the MZI phase shift and its amplification factor is given by $\Delta \phi(t)=$ $-(\alpha / 2) \ln G_{1}(t)$. The shape of the output pulse is determined by the time dependence of $G_{1}(t)$ both directly and through $\Delta \phi(t)$ according to equation (11) resulting in a Gaussian doublet under certain conditions determined by the QD SOA dynamics that will be discussed in section 3 . 


\section{Ultra-fast Nonlinear Phenomena in QD SOA}

In this section, we will discuss the structure and operation principles of QD SOA. We briefly review the QD specific properties, energy bands, and electron transitions responsible for the stimulated light emission and amplification in subsection 3.1. Theory of QD SOA operation based on the electron rate equations and photon propagation equation has been developed in a large number of works. See, for example, Berg (2004), Qasaimeh (2003), Qasaimeh (2004), Ben Ezra (September 2005), Ben Ezra (October 2005), Ben Ezra (2007) and references therein. It is considered in subsections 3.2 and 3.3.

\subsection{Structure and Operation Principles of QD SOA}

Quantization of of electron states in all three dimensions results in a creation of a novel physical object - a macroatom, or quantum dot (QD) containing a zero dimensional electron gas. Size quantization is significantly effective when the quantum dot three dimensions are of the same order of magnitude as the electron de Broglie wavelength Ustinov (2003). Detailed theoretical and experimental investigations of InAs/GaAs and InAs QDs electronic structure taking into account their shape (lens-shaped, pyramidal), size, composition profile, and production technique (Stranski-Krastanow, colloidal) have been carried out Bimberg (1999), Bányai (2005), Ustinov (2003). The different types of QDs based on different technologies and operating in different parts of spectrum are known such as $\operatorname{In}(\mathrm{Ga})$ As QDs grown on GaAs substrates, InAs QDs grown on InP substrates, and colloidal free-standing InAs QDs. In(Ga)As/GaAs QDs are characterized by emission at wavelengths no longer than $\lambda=1.35 \mu \mathrm{m}$, while the InAs/InP structures have been proposed for emission at the usual telecommunication wavelength $\lambda=1.55 \mu \mathrm{m}$.

A system of QDs can be approximated with a three energy level model in the conduction band containing a spin degenerate ground state GS, fourfold degenerate excited state (ES) with comparatively large energy separations of about $50-70 \mathrm{meV}$, and a narrow continuum wetting layer (WL). The electron WL is situated $150 \mathrm{meV}$ above the lowest electron energy level in the conduction band, i.e. GS and has a width of approximately $120 \mathrm{meV}$. In real cases, the QDs vary in size, shape, and local strain which leads to the fluctuations in the quantized energy levels and the inhomogeneous broadening in the optical transition energy. A Gaussian distribution may be used for the description of the QD sizes, and it shows that the discrete resonances merge into a continuous structure with widths around 10\% Bányai (2005). The QDs and WL are surrounded by a barrier material which prevents direct coupling between QD layers. The absolute number of states in the WL is much larger than in the QDs. GS and ES in QDs are characterized by homogeneous and inhomogeneous broadening Bányai (2005). The homogeneous broadening caused by the scattering of the optically generated electrons and holes with imperfections, impurities, phonons, or through the radiative electron-hole pair recombination Bányai (2005) is about $15 \mathrm{meV}$ at room temperature.

The active region of a QD SOA is a layer including self-assembled InGaAs QDs on a GaAs substrate Sugawara (2004). Typically, the QD density per unit area is about $\left(10^{10}-10^{11}\right) \mathrm{cm}^{-2}$. The bias current is injected into the active layer including QDs, and the input optical signals are amplified via the stimulated emission or processed via the optical nonlinearity by QDs Sugawara (2004). The stimulated radiative transitions occur between GS and the valence band of QDs. A detailed theory of QD SOAs based on the density matrix approach has been developed in the pioneering work Sugawara (2004) where the linear and nonlinear optical responses of QD SOAs with arbitrary spectral and spatial distribution of quantum dots in active 
region under the multimode light propagation have been considered. It has been shown theoretically that XGM takes place due to the coherent terms under the condition that the mode separation is comparable to or less than the polarization relaxation rate $\left|\omega_{m}-\omega_{n}\right| \leq \Gamma_{g}$ where $\omega_{m, n}$ are the mode frequencies and the relaxation time $\tau=\Gamma_{g}^{-1}=130 \mathrm{fs}_{s}$ Sugawara (2004). XGM is also possible in the case of the incoherent nonlinear polarization, or the so-called incoherent spectral hole burning Sugawara (2004). It has been assumed that XGM occurred only for signals with a detuning limited by the comparatively small homogeneous broadening, and for this reason the ensemble of QDs should be divided into groups by their resonant frequency of the GS transition between the conduction and valence bands Sugawara (2004).

\subsection{Rate Equations and Dynamics of QD SOA}

The phenomenological approach to the QD SOA dynamics is based on the rate equations for the electron densities of GS, ES and for combined WL and barrier serving as a reservoir. It is determined by electrons, because of the much larger effective mass of holes and their smaller state spacing Berg (2004). The carrier dynamics is characterized by slow relaxation processes between WL and ES, and the rapidly varying coherent nonlinear population terms vanish after the averaging over the comparatively large relaxation time $\tau_{w 2} \sim$ several ps from the two-dimensional WL to the ES. We have taken into account only incoherent population terms because for XGM between modes with the maximum detuning $\Delta \lambda_{\max }=30 \mathrm{~nm}$ within the especially important in optical communications conventional band (C-band) of $\lambda=(1530 \div 1565) n m$ the condition $\omega_{1}-\omega_{2}>\Gamma_{g}^{-1}$ is valid even for the lowest relaxation time from the ES to GS $\tau_{21}=0.16 p s$, and the rapidly varying coherent beating terms are insignificant Sugawara (2004). The direct carrier capture into the GS is neglected due to the fast intradot carrier relaxation and the large energy separation between the GS and the WL and it is assumed that the charge neutrality condition in the GS is valid. The rate equations account for both the fast transitions form WL to ES and GS and the slow dynamics of the spontaneous transitions and electron escape from ES back to WL Qasaimeh (2003), Qasaimeh (2004), Ben Ezra (2007). They have the form Qasaimeh (2003), Qasaimeh (2004), Ben Ezra (2007).

$$
\begin{gathered}
\frac{\partial N_{w}}{\partial t}=\frac{J}{e L_{w}}-\frac{N_{w}(1-h)}{\tau_{w 2}}+\frac{N_{w} h}{\tau_{2 w}}-\frac{N_{w}}{\tau_{w R}}, \\
\frac{\partial h}{\partial t}=\frac{N_{w} L_{w}(1-h)}{N_{Q} \tau_{w 2}}-\frac{N_{w} L_{w} h}{N_{Q} \tau_{2 w}}-\frac{(1-f) h}{\tau_{21}}+\frac{f(1-h)}{\tau_{12}}, \\
\frac{\partial f}{\partial t}=\frac{(1-f) h}{\tau_{21}}-\frac{f(1-h)}{\tau_{12}}-\frac{f^{2}}{\tau_{1 R}} \\
-\frac{g_{p} L}{N_{Q}}(2 f-1) S_{p} \frac{c}{\sqrt{\varepsilon_{r}}}-\frac{g_{s} L}{N_{Q}}(2 f-1) S_{s} \frac{c}{\sqrt{\varepsilon_{r}}} .
\end{gathered}
$$

Here, $S_{p}, S_{s}$ are the CW pump and on-off-keying (OOK) modulated signal wave photon densities, respectively, $L$ is the length of SOA, $g_{p}, g_{s}$ are the pump and signal wave modal gains, respectively, $f$ is the electron occupation probability of GS, $h$ is the electron occupation probability of ES, $e$ is the electron charge, $t$ is the time, $\tau_{2 w}$ is the electron escape time from the ES to the $\mathrm{WL}, \tau_{w R}$ is the spontaneous radiative lifetime in $\mathrm{WL}, \tau_{1 R}$ is the spontaneous radiative lifetime in QDs, $N_{Q}$ is the surface density of QDs, $N_{w}$ is the electron density in the WL, $L_{w}$ is the effective thickness of the active layer, $\tau_{21}$ is the electron relaxation time from the ES to GS and 
$\tau_{12}$ is the electron relaxation time from the GS to the ES, $\varepsilon_{r}$ is the SOA material permittivity, $c$ is the velocity of light in free space.The modal gain $g_{p, s}(\omega)$ is given by Uskov (2004)

$$
g_{p, s}(\omega)=\frac{2 \Gamma N_{Q}}{a} \int d \omega F(\omega) \sigma\left(\omega_{0}\right)(2 f-1)
$$

where the number $l$ of QD layers is assumed to be $l=1$, the confinement factor $\Gamma$ is assumed to be the same for both the signal and the pump waves, $a$ is the mean size of QDs, $\sigma\left(\omega_{0}\right)$ is the cross section of interaction of photons of frequency $\omega_{0}$ with carriers in QD at the transition frequency $\omega$ including the homogeneous broadening factor, $F(\omega)$ is the distribution of the transition frequency in the QD ensemble which is assumed to be Gaussian Qasaimeh (2004), Uskov (2004). It is related to the inhomogeneous broadening and it is described by the expression Uskov (2004)

$$
F(\omega)=\frac{1}{\Delta \omega \sqrt{\pi}} \exp \left[-\frac{(\omega-\bar{\omega})^{2}}{(\Delta \omega)^{2}}\right]
$$

where the parameter $\Delta \omega$ is related to the inhomogeneous linewidth $\gamma_{i n \text { hom }}=2 \sqrt{\ln 2} \Delta \omega$, and $\bar{\omega}$ is the average transition frequency.

\subsection{XGM and XPM in QD SOA}

In order to describe adequately XGM and XPM in QD SOA we should take into account the interaction of QDs with optical signals. The optical signal propagation in a QD SOA is described by the following truncated equations for the slowly varying $\mathrm{CW}$ and pulse signals photon densities and phases $S_{C W, P}=P_{C W, P} /\left(\hbar \omega_{C W, P}\left(v_{g}\right)_{C W, P} A_{e f f}\right)$ and $\theta_{C W, P}$ Agrawal (1989).

$$
\begin{gathered}
\frac{\partial S_{C W, P}(z, \tau)}{\partial z}=\left(g_{C W, P}-\alpha_{i n t}\right) S_{C W, P}(z, \tau) \\
\frac{\partial \theta_{C W, P}}{\partial z}=-\frac{\alpha}{2} g_{C W, P}
\end{gathered}
$$

Here $P_{C W, P}$ are the CW and pulse signal optical powers, respectively, $A_{e f f}$ is the QD SOA effective cross-section, $\omega_{C W, P},\left(v_{g}\right)_{C W, P}$ are the $\mathrm{CW}$ and pulse signal group angular frequencies and velocities, respectively, $g_{C W, P}$ are the active medium (SOA) gains at the corresponding optical frequencies, $\alpha_{i n t}$ is the absorption coefficient of the SOA material. For the pulse propagation analysis, we replace the variables $(z, t)$ with the retarded frame variables $\left(z, \tau=t \mp z / v_{g}\right)$. For optical pulses with a duration $T \gtrsim 10 p s$ the optical radiation of the pulse is filling the entire active region of a QD SOA of the length $L \lesssim 1 \mathrm{~mm}$ and the propagation effects can be neglected Gehrig (2002). Hence, in our case the photon densities

$$
S_{C W, P}(z, \tau)=\left(S_{C W, P}(\tau)\right)_{i n} \exp \left[\int_{0}^{z}\left(g_{C W, P}-\alpha_{i n t}\right) d z^{\prime}\right]
$$

can be averaged over the QD SOA length $L$ which yields

$$
S_{C W, P}(\tau)=\frac{1}{L}\left(S_{C W, P}(\tau)\right)_{\text {in }} \int_{0}^{L} d z \exp \left[\int_{0}^{z}\left(g_{C W, P}-\alpha_{i n t}\right) d z^{\prime}\right]
$$


Solution of equation (18) yields for the phases which should be inserted into MZI equation (11)

$$
\theta_{C W, P}(\tau)=-(\alpha / 2) \int_{0}^{L} d z g_{C W, P} .
$$

The time-dependent variations of the carrier distributions in the QDs and WL result in the strong phase changes (18) during the light propagation in the QD SOA Gehrig (2002). System of equations (12)-(14) with the average pump and signal photon densities (20) and phases (21) constitutes a complete set of equations describing XGM and XPM in QD SOA related by the LEF $\alpha$ as it is seen from equations (17), (18) and (21).

\section{Simulation Results for IR UWB Generation in MZI with QD SOA}

The analytical solution of nonlinear and extremely complicated equations (12)-(14) in a closed form is hardly possible, and for this reason, the system of these equations has been solved numerically for the following typical values of the QD SOA parameters Ben Ezra (2007), Ben Ezra (2008). The QD SOA active region length $L$ and width $W$ are, respectively, $L=2 \mathrm{~mm}$, $W=10 \mu \mathrm{m}$; the confinement factor $\Gamma=3 \times 10^{-2}$, the maximum gain $g_{\max }=11.5 \mathrm{~cm}^{-1}$, $\alpha_{i n t}=3 \mathrm{~cm}^{-1}$, the fastest relaxation time for the transitions between ES and GS is $\tau_{21}=0.16 \mathrm{ps}$, while the largest relaxation time for the transition between ES and WL is about $\tau_{2 w} \sim 1 n s$. The simulation results for the output power Gaussian doublet and temporal variations of the optical signal power and phase in upper and lower arms of MZI are shown in Fig. 4.
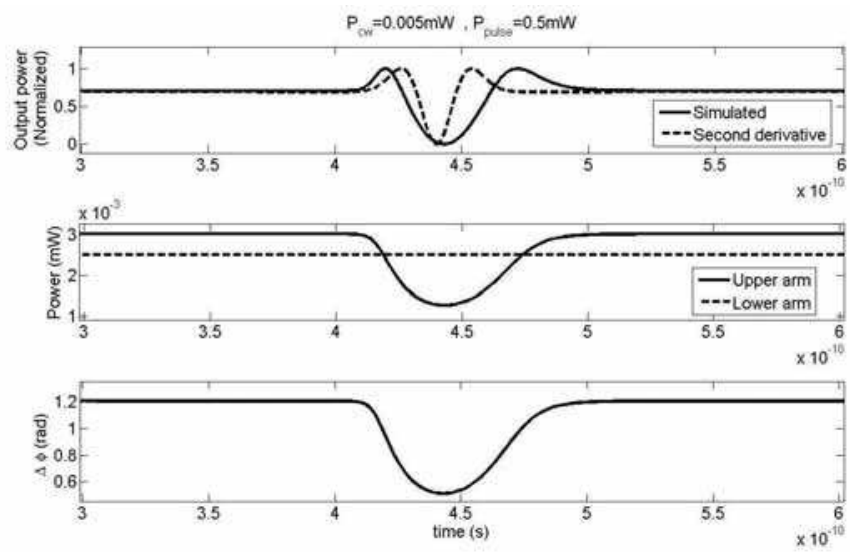

Fig. 4. Gaussian doublet of the output power pulse (solid line) and the second derivative of the Gaussian pulse (dashed line) for the pulsed laser optical power of $P_{p}=0.5 \mathrm{~mW}$ (upper box); optical signal powerin the upper arm of MZI (middle box); phase difference $\Delta \phi$ (lower box)

At high Gaussian pulse power levels QD SOA passes to the nonlinear saturation regime where the amplification factor $G_{1}(t)$ and XPM phase shift $\Delta \phi(t)$ decrease to their minimum values. In this case, $P_{\text {out }}$ also reaches its lowest level due to the maximum value of the oscillating term in equation (11). In such a case the XPM process is dominant, and the Gaussian doublet occurs as it is seen from Fig.4. The local maxima of the doublet can be explained by the dominant 

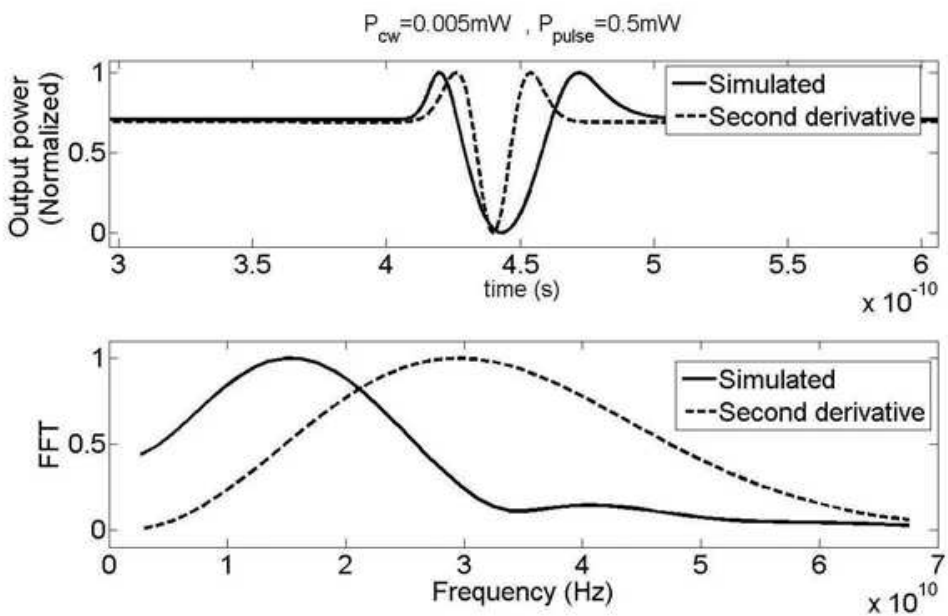

Fig. 5. Simulated Gaussian doublet (solid line) and the second derivative the standard Gaussian pulse (dashed line (upper box); the spectra (FFTs) of the simulated IR UWB pulse (solid line) and second derivative of the standard Gaussian pulse (dashed line) (lower box)

role of XGM process in the regions of small variations of $\Delta \phi(t)$. In such a case, the variation of the oscillating term in equation (11) is negligible. This term is varying more slowly as compared to $G_{1}(t)$ because of the logarithmic form of the argument $\Delta \phi(t)$, except for the QD SOA saturation regime where $G_{1}(t)$ tends to its minimum value.

The simulated IR UWB doublet, second derivative of the standard Gaussian pulse and their fast Fourier transforms (FFTs), i.e. spectra are shown in Fig. 5. The spectrum of the simulated IR UWB signal manifests the filtering features of the proposed IR UWB generator Ghawami (2005). For the Gaussian pulse duration of about tens of picoseconds, the filtering behavior of the proposed IR UWB generator is caused by the fast transition relaxation time between GS and ES $\tau_{12} \sim 1 p s$ in QD SOA limiting a rise time and a fall time of the pulse propagating through QD SOA. The operation rate of QD SOA is also strongly influenced by the SOA bias current and optical power Sugawara (2004), Ben Ezra (2007).

\section{All-optical Passive Generation of IR UWB}

UWB pulse generation techniques in optical domain discussed above are characterized by complicated and costly systems combining electronic and active nonlinear photonic elements such as SOA which are necessary for mixing and conversion of UWB modulated optical carriers with different optical frequencies. We propose an alternative method of all-optical generation of UWB Gaussian monocycles and doublets based on the system of two integrated unbalanced MZIs (UMZIs) connected in parallel that does not contain any active elements. The UMZIs are chosen in such a way that the phase difference of the interfering signals at the output is equal to $\pi$. Each one of the UMZIs is characterized by time delay differences $\tau$ and $2 \tau$. As a result, at the output of the UMZIs the interfering optical signals UWB modulated by a Gaussian UWB pulse form the first-order difference approximating the Gaussian monocycle and the second-order difference approximating the Gaussian doublet. At the output of the system the UWB monocycles and doublets can be converted to a UWB signal by means 
of a homodyne detection where the local oscillator frequency $\omega_{L O}$ coincides with the optical carrier frequency $\omega$ Agrawal (2002). In such a case the detected signal is proportional to the UWB modulated optical signal amplitude Agrawal (2002), i.e. to the Gaussian monocycle or doublet in our case. The system can be made tunable by including into UMZIs a tunable delay line.

The block diagram of the UMZIs is shown in Fig. 6. The UWB modulated optical carrier field at the system input is given by

$$
E(t)=A(t) \exp [-i(\omega t+\phi)]
$$

where $A(t)$ and $\phi$ are the amplitude and phase of the input signal, respectively. The signal (22) is split in the in the two equal parts at the input of the system, and then the each signal is split equally once more at the input of the both UMZIs. Assuming the delay time $\tau$ of the lower arm of the upper UMZI1 and of the upper arm of the lower UMZI2 and the delay time $2 \tau$ of the lower arm of the lower UMZI2 we can describe the interfering optical fields as follows.

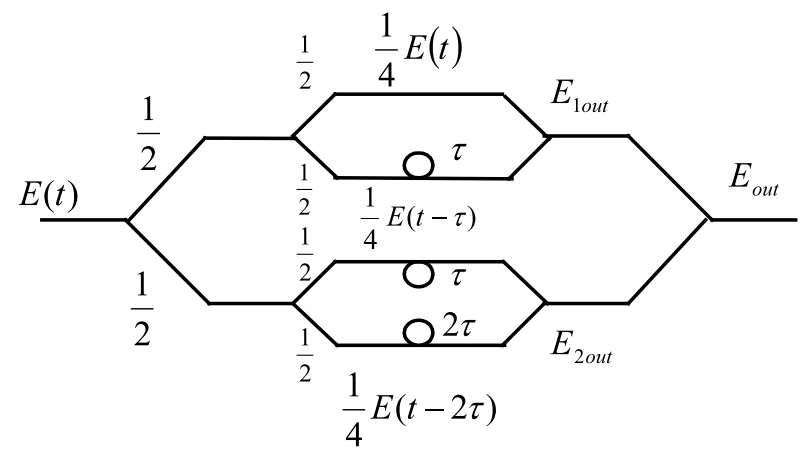

Fig. 6. The UMZIs connected in parallel

$$
\begin{gathered}
E_{1,2 \text { in }}=\frac{1}{2} E(t) ; E_{1 \text { up }}=\frac{1}{4} E(t) ; E_{1 \text { low }}=\frac{1}{4} E(t-\tau) \\
E_{1 \text { out }}=E_{1 \text { up }}-E_{1 \text { low }}=\frac{1}{4}[E(t)-E(t-\tau)] \\
E_{2 \text { up }}=\frac{1}{4} E(t-\tau) ; E_{2 \text { low }}=\frac{1}{4} E(t-2 \tau) \\
E_{2 \text { out }}=E_{2 \text { up }}-E_{2 \text { low }}=\frac{1}{4}[E(t-\tau)-E(t-2 \tau)] \\
E_{\text {out }}=E_{1 \text { out }}-E_{2 o u t}=\frac{1}{4}[E(t)-2 E(t-\tau)+E(t-2 \tau)]
\end{gathered}
$$

Equations (24)-(27) show that the output fields $E_{1 o u t}, E_{2 o u t}$ of the UMZI1 and UMZI2, respectively, correspond to the first-order difference of the input signal $E(t)$, and the system output signal $E_{\text {out }}$ corresponds to the second-order difference of $E(t)$ which represent the approximations of the first and second derivative of $E(t)$, respectively Yao (2007). In the case of the Gaussian UWB modulated input signal, the first derivative is the monocycle and second derivative is the doublet Ghawami (2005). In order to down-convert the output monocycle and doublet modulated optical signals, the coherent-detection technique is used where the 
local oscillator frequency $\omega_{L O}=\omega$ Agrawal (2002). In order to improve the accuracy of the homodyne detection we use the same laser as a source of the externally UWB modulated optical signal and the CW optical signal. The block diagram of the system is shown in Fig. 7. The detector photocurrent $I(t)$ is given by Agrawal (2002)

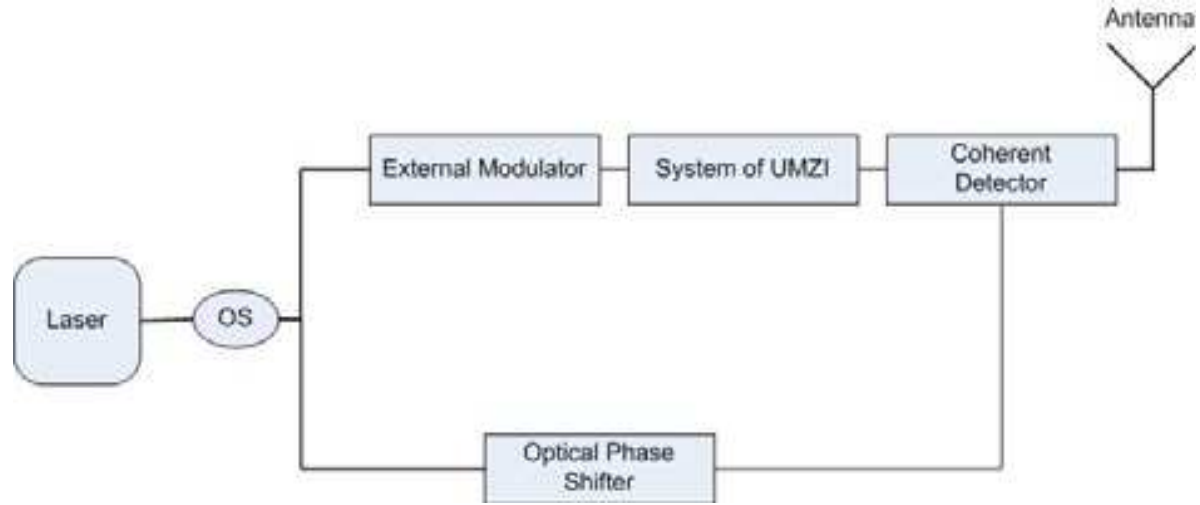

Fig. 7. Block diagram of UWB pulse photonic generation system

$$
I(t)=R\left[P_{\text {out }}+P_{L O}+2 \sqrt{P_{\text {out }} P_{L O}} \cos \left(\phi_{\text {out }}-\phi_{L O}\right)\right]
$$

where $R$ is the detector responsivity, $P_{\text {out }}=K A_{\text {out }}^{2}, P_{L O}=K A_{L O}^{2}, A_{\text {out }}, A_{L O}, \phi_{\text {out }}, \phi_{L O}$ are the output signal and local oscillator optical powers, amplitudes and phases, respectively, $K$ is a constant of proportionality. The phase difference $\left(\phi_{\text {out }}-\phi_{L O}\right)$ can be eliminated by using a phase shifter. Then, the detected signal $I_{H}(t)$ is given by Agrawal (2002)

$$
I_{H}(t)=2 R \sqrt{K P_{L O}} A_{\text {out }} \sim\left|E_{\text {out }}\right|
$$

Equations (27) and (29) show that the detected signal $I_{H}(t)$ is proportional to the Gaussian doublet. Typically, the local oscillator power is much larger than the output signal, and optical losses in the MZI system can be neglected. For the typical values of $R=(0.4-0.9) \mathrm{A} / \mathrm{W}$ Agrawal (2002), $P_{L O}=10 \mathrm{~mW}, P_{\text {out }}=0.1 \mathrm{~mW}$ we obtain $I_{H}(t) \approx(0.8-1.8) \mathrm{mA}$.

The numerical simulations results for equations (24)-(27) are shown in Figs. 8, 9.

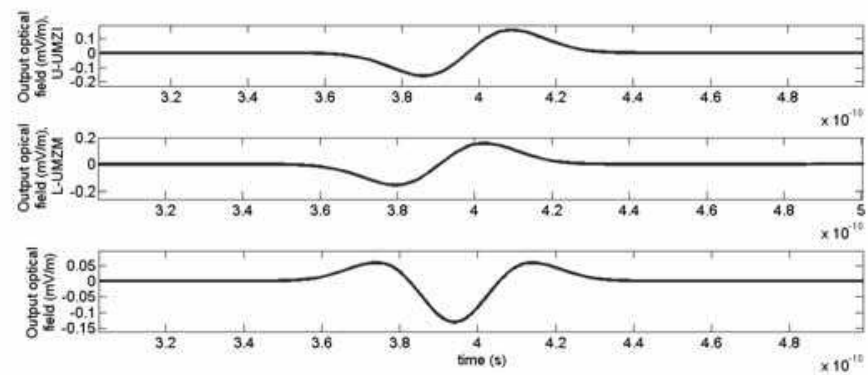

Fig. 8. Gaussian monocycles (the upper and middle boxes) and doublet (the lower box) 


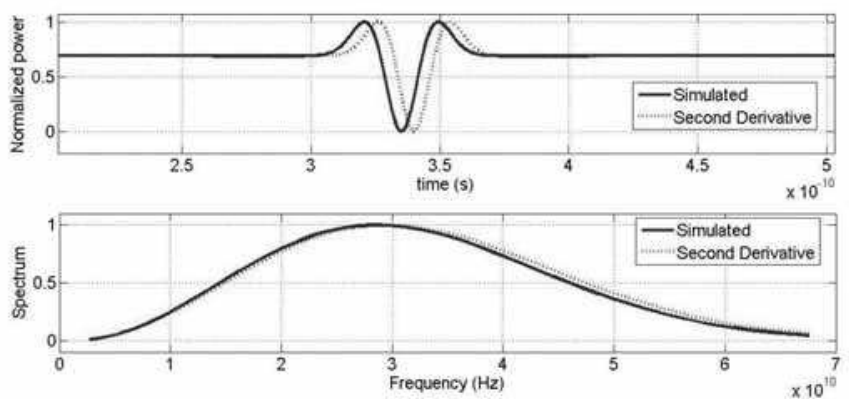

Fig. 9. Simulated Gaussian doublet at the output of the passive UNMZI system - solid line, the second derivative of Gaussian pulse - dashed line (the upper box); the spectra of the Gaussian doublet (solid line) and of the second derivative of Gaussian pulse (dashed line) (the lower box)

As it is seen from Fig. 8, the Gaussian monocycles and doublets can be optically generated by using the system of the passive UMZIs connected in parallel and coherent homodyne detector. Fig. 9 shows a satisfactory coincidence between the simulated Gaussian doublet and its spectrum and the calculated second derivative of the standard Gaussian pulse (3) and its spectrum.

Unlike the existing methods, the proposed method is based on the generation of the field pulses instead of the power pulses. The advantages of the proposed method are mainly related to the absence of the nonlinear active elements which results in the stability, simplicity and easy control of the proposed system.

\section{All-optical Processing of UWB Signals}

A wavelet transform (WT) involves time-frequency representation of nonstationary signals and can be successfully used in optical communications for signal processing operations such as an ultrafast image transmission, signal denoising, time-frequency multiplexing, spectral and temporal encoding Cincotti (2005). The use of WTs in optical communications can significantly improve a system capacity due to the two-dimensional processing capability of the multiresolution analysis (MRA) providing a high resolution at low frequency and low resolution at high frequency and permitting to localize a particular portion of the signal Cincotti (2005). The continuous WT (CWT) of a signal $s(t)$ is given by Rao (1998)

$$
\operatorname{CWT}_{S}(a, \tau)=\frac{1}{|a|} \int s(t) \Psi^{*}\left(\frac{t-\tau}{a}\right) d t
$$

where $a$ is a real scale parameter, $\tau$ is the translation factor, and $\psi(t)$ is the mother wavelet function. In digital signal processing and multiplexing the orthogonal wavelet series expansions or discrete WTs (DWTs) are more useful that can be obtained from CWT (30) when the scale and translation factors have discrete values. DWT decomposes the input signal at different scales and resolutions using bases localized in time and frequency domains and extracting specific information from the signal. Under certain conditions, a signal $s(t)$ can be represented 
by a smoothed approximation at resolution $2^{M}$ given by Cincotti (2005)

$$
s(t)=\sum_{k} 2^{-M / 2} c_{M}[k] \phi\left(2^{-M} t-k \Delta \tau\right)+\sum_{l=1}^{M} \sum_{k} 2^{-l / 2} d_{l}[k] \psi\left(2^{-l} t-k \Delta \tau\right)
$$

where $\psi(t)$ is the mother wavelet function, $\phi(t)$ is the scaling function, $c_{M}[k]$ are the scaling/approximation coefficients, $d_{l}[k]$ the detail coefficients, and $\Delta \tau$ is the inverse of the signal free spectral range (FSR). The DWT decomposition (31) halves the time resolution and doubles the frequency band of original signal. In the case of the signal corruption by white Gaussian noise, the signal denoising scheme is based on the selective wavelet reconstruction since an inhomogeneous signal is described by a small number of wavelet coefficients while the white noise is distribute over a large number of coefficients Cincotti (2005).

Passive networks for a physical implementation of the discrete WT (DWT) can be based on passive MZIs described in section 5 with output asymmetric couplers Cincotti (2002). We propose a passive UMZI as a basic component for the all-optical passive generation of the Mexican hat mother wavelet (MHMW) and for the realization of the Haar wavelet decomposition which can be used for the all-optical signal denoising.

Using the system of passive UMZIs shown in Fig. 6 with the time delay $\Delta \tau$ instead of $\tau$ and applying equations (23)-(27) to the Gaussian input signal we indeed obtain the approximation of the Gaussian second derivative which is MHMW given by Rao (1998)

$$
E(t)=\left(1-t^{2}\right) \exp \left(-t^{2}\right)
$$

The inverse MHMW corresponds to the doublet shown in Fig. 8.

Consider now the all-optical signal denoising. It is based on the Haar recursive decomposition with the quadrature mirror filter (QMF) coefficients $h[k]$ and $g[k]$. These coefficients can be realized by the digital filters given by

$$
h[k]=\frac{1}{2}[\delta(k)+\delta(k+1)] ; g[k]=\frac{1}{2}[\delta(k)-\delta(k+1)]
$$

These filters can be implemented by the UMZI. In the case of low pass filter $h[k]$ the interference at the UMZI output should be constructive. In the opposite case of the high pass filter $g[k]$, the interference at the UMZI output should be destructive. We generated a noisy $10 \mathrm{~Gb} / \mathrm{s}$ NRZ optical signal. The eye diagram of this input signal is shown in Fig. 10. In order to carry out the denoising of the input NRZ signal we used a three stage cascade of the UMZIs of the type shown in Fig. 6 with FSR of $\Delta \tau, 2 \Delta \tau, 3 \Delta \tau$, respectively, which permits the realization of the three level Haar decomposition of the input signal. By eliminating the higher order detail components $d_{l}[k]$ we filter the noise components and simultaneously slightly reshape the input waveform itself. The eye diagram of the denoised signal is shown in Fig. and manifests a substantial improvement of the signal-to-noise ratio (SNR).

\section{Conclusions}

We have proposed a theoretical analysis of novel all-optical methods of the IR UWB signal generation.

1. We proposed a novel method of the IR UWB signal generation based on integrated MZI with a QD SOA as a nonlinear component. The generation mechanism is related to 


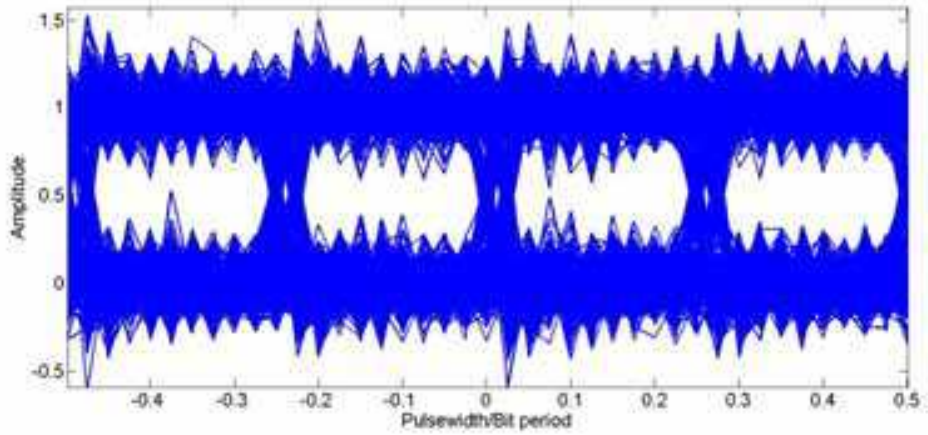

Fig. 10. The eye diagram of the input optical signal

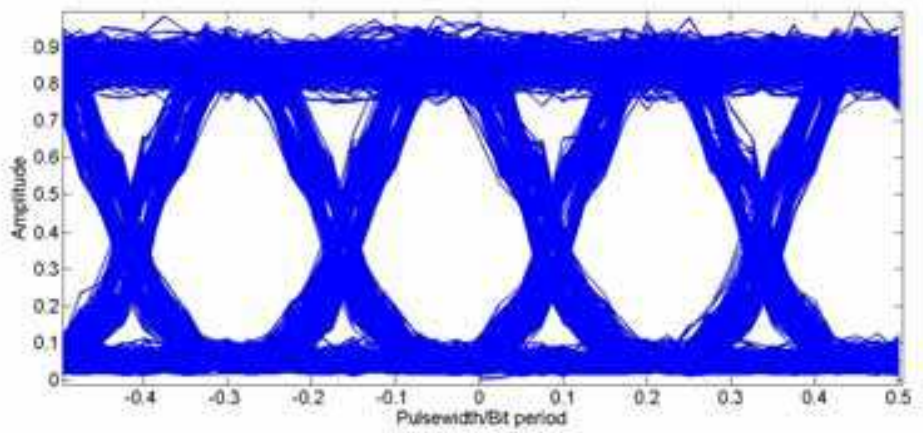

Fig. 11. The eye diagram of the optical signal after the wavelet denoising process

XPM and XGM phenomena in QD SOA Unlike other proposed all-optical methods, we need not optical fibers, FBG and EOM substantially reducing the cost and complexity of a IR UWB generator. The IR UWB signals generated by the proposed QD SOA based MZI structure have the form of the Gaussian doublet. The shape of the signal and its spectrum can be tailor-made for different applications by changing the QD SOA bias current and optical power.

2. We have shown theoretically that the Gaussian monocycles and doublets can be optically generated by using the system consisting of the passive UMZIs connected in parallel, and coherent homodyne detector. Unlike the existing methods, the proposed method is based on the generation of the field pulses instead of the power pulses and does not require nonlinear components.

3. We proposed the implementation scheme of the all-optical signal denoising and MHMW generation based on the system of the passive UMZIs. 


\section{References}

Agrawal, G.P. \& Olsson, N.A. (1989). Self-phase modulation and spectral broadening of optical pulses in semiconductor laser amplifiers. IEEE Journal of Quantum Electronics, Vol. 25, No.11, (November 1989) 2297-2306, ISSN 0018-9197

Agrawal, G.P. (2001). Applications of Nonlinear Fiber Optics. Academic Press, ISBN 0-12-0451441 , New York

Agrawal, G.P. (2002). Fiber-Optic Communication Systems. Wiley, ISBN 0-471-21571-6, New York

Bányai, L. \& Koch, S. W. (2005). Semiconductor Quantum Dots (Second Edition). World Scientific, ISBN 9810213905, London,

Ben-Ezra, Y.; Haridim, M. \& Lembrikov, B. I. (2005). Theoretical analysis of gain-recovery time and chirp in QD-SOA. IEEE Photonics Technology Letters, Vol. 17, No. 9, (September 2005) 1803-1805, ISSN 1041-1135

Ben-Ezra, Y.; Lembrikov, B. I. \& Haridim, M. (2005). Acceleration of gain recovery and dynamics of electrons in QD-SOA. IEEE Journal of Quantum Electronics, Vol. 41, No. 10, (October 2005) 1268-1273, ISSN 0018-9197

Ben-Ezra, Y.; Lembrikov, B. I. \& Haridim, M. (2007). Specific features of XGM in QD-SOA. IEEE Journal of Quantum Electronics, Vol.43, No. 8, (August 2007) 730-737, ISSN 00189197

Ben Ezra, Y.; Haridim, M.; Lembrikov, B.I. \& Ran, M. (2008). Proposal for All-optical Generation of Ultra Wideband Impulse Radio Signals in Mach-Zehnder Interferometer with Quantum Dot Optical Amplifier. IEEE Photonics Technology Letters, Vol. 20, No. 7 (April 2008) 484-486, ISSN 1041-1135

Berg, T.W.; Mørk, J. \& Hvam, J.M. (2004). Gain dynamics and saturation in semiconductor quantum dot amplifiers. New Journal of Physics, Vol. 6, No. 178, (2004) 1-23, ISSN $1367-2630$

Bimberg, D.; Grundmann, M. \& Ledentsov, N. N. (1999). Quantum Dot Heterostructures. John Wiley, ISBN 047 1973882, New York

Chen, H.; Zhu, G.; Wang, Q.; Jaques, J.; Leuthold, J.; Picirilli, A.B. \& Dutta, N.K. (2002). Alloptical logic XOR using differential scheme and Mach-Zender interferometer. Electronic Letters, Vol. 38, No. 21, (October 2002) 1271-1273, ISSN 0013-5194

Chen, H.; Chen, M.; Wang, T.; Li, M. \& Xie, S. (2008). Methods for ultra-wideband pulse generation based on optical cross-polarization modulation. Journal of Lightwave Technology, Vol. 26, No. 15, (August 2008) 2492-2499, ISSN 0733-8724

Cincotti, G. (2002). Fiber Wavelet Filters. IEEE Journal of Quantum Electronics, Vol. 38, No. 10, (October 2002) 1420-1427, ISSN 0018-9197

Cincotti, G.; Moreolo, M.S. \& Neri, A. (2005). Optical Wavelet Signals Processing and Multiplexing. EURASIP Journal on Applied Signal Processing, Vol. 2005, (January 2005) 15741583, ISSN 1110-8657

Dong, J.; Zhang, X. \& Huang (2009). All-optical ultra-wideband pulse generation based on semiconductor optical amplifiers. Frontiers of Optoelectronics in China, Vol. 2, No.1, (March 2009) 40-49, ISSN 1674-4128

E. Gehrig, E. \& O. Hess, O. (2002). Mesoscopic spatiotemporal theory for quantum-dot lasers. Phys. Rev. A, Vol. 65, No. 3, (March 2002) 033804-1-16, ISSN 1050-2947

Ghavami, M.; Michael, L.B. \& Kohno, R. (2005). Ultra Wideband Signals and Systems in Communication Engineering, Wiley, ISBN-10 0-470-86571-5(H/B), Chichester, England

Joergensen, C. ; Danielsen, S.L.; Durhuus, T.; Mikkelsen, B. ; Stubkjaer, K.E.; Vodjdani, N.; Ratovelomanana, F.; Enard, A.; Glastre, G.; Rondi, D. \& Blondeau, R. (1996). Wave- 
length conversion by optimized monolithic integrated Mach-Zender interferometer, IEEE Photonics Technology Letters, Vol. 8, No. 4, (April 1996) 521-523, ISSN 1041-1135

Kanellos, G.T.; Petrantonakis, D.; Tsiokos, D.; Bakopoulos, P.; Zakynthinos, P.; Pleros, N.; Apostolopoulos, D.; Maxwell, G.; Poustie, A. \& Avramopoulos, H. (2007). All-optical 3R burst-mode reception at $40 \mathrm{~Gb} / \mathrm{s}$ using four integrated MZI switches. Journal of Lightwave Technology, Vol. 25, No. 1, (January 2007) 184-192, ISSN 0733-8724

Kshetrimayum, R. S. (2009). An Introduction to UWB Communication Systems. Potentials, IEEE, Vol. 28, Issue 2, (March-April 2009) 9-13, ISSN 0278-6648

Le Guennec, Y. \& Gary, R. (2007). Optical frequency conversion for millimeter-wave ultrawideband-over-fiber systems. IEEE Photonics Technology Letters, Vol. 19, No. 13, (July 2007) 996-998, ISSN 1041-1135

Lin, W.-P. \& Chen, J.-Y. (2005). Implementation of a new ultrawide-band impulse system, IEEE Photonics Technology Letters, Vol. 17, No. 11, (November 2005) 2418-2420, ISSN 10411135

Qasaimeh, O. (2003). Optical gain and saturation characteristics quantum-dot semiconductor optical amplifiers. IEEE J. of Quantum Electronics, Vol. 39, No. 6, (June 2003) 793-798, ISSN 0018-9197

Qasaimeh, O. (2004). Characteristics of cross-gain (XG) wavelength conversion in quantum dot semiconductor optical amplifiers. IEEE Photonics Technology Letters, Vol. 16, No. 2, (February 2004) 542-544, ISSN 1041-1135

Ran, M; Ben Ezra, Y. \& Lembrikov B.I. (2009). Ultra-wideband Radio-over-optical-fibre Technologies, In Short-Range Wireless Communications, Kraemer, R. \& Katz, M. D. (Eds.), 271-327, Wiley, ISBN 978-0-470-69995-9 (H/B), Chichester, England

Rao, R.M. \& Bopardikar, A.S. (1998). Wavelet Transforms. Introduction to Theory and Applications. Addison-Wesley, ISBN-10: 0201634 635, Reading, Massachusetts

Sugawara, M.; Ebe, H.; Hatori, N.; Ishida, M.; Arakawa, Y.; Akiyama, T.; Otsubo, K. \& Nakata, Y. (2004) Theory of optical signal amplification and processing by quantum-dot semiconductor optical amplifiers. Phys.Rev.B, Vol. 69, No. 23 (June 2004) 235332-1-39, ISSN 1098-0121

Uskov, A.V. ; Berg, T.W. \& Mørk, J. (2004). Theory of pulse-train amplification without patterning effects in quantum-dot semiconductor optical amplifiers. IEEE J. of Quantum Electronics, Vol. 40, No. 3, (March 2004) 306-320, ISSN 0018-9197

Ustinov, V.M.; Zhukov, A.E.; Egorov, A. Yu. \& Maleev, N. A. (2003). Quantum Dot Lasers, Oxford University Press, ISBN 019852679 2, Oxford

Wang, Q.; Zhu, G.; Chen, H. ; Jaques, J. ; Leuthold, J.; Picirilli, A.B. \& Dutta, N.K. (2004). Study of all-optical XOR using Mach-Zehnder interferometer and differential scheme. IEEE J. of Quant. Electr., Vol. 40, No. 6, (June 2004) 703-710, ISSN 0018-9197

Wang, Q. \& Yao, J. (2006). UWB doublet generation using nonlinearly-biased electro-optic intensity modulator, Electronic Letters, Vol. 42, No. 22, (October 2006)1304-1305, ISSN 0013-5194

Yang, L. \& Giannakis, G.B. (2004). Ultra-Wideband Communications. IEEE Signal Processing Magazine, Vol. 21, No. 6; (November 2004) 26-54, ISSN 1053-5888

Yao, J.; Zeng, F. \& Wang, Q. (2007). Photonic generation of ultrawideband signals. Journal of Lightwave Technology, Vol. 25, No. 11, (November 2007) 3219-3235, ISSN 0733-8724

Zeng, F. \& Yao, J. (2006). An approach to ultrawideband pulse generation and distribution over optical fiber. IEEE Photonics Technology Letters, Vol. 18, No. 7, (April 2006) 823825, ISSN 1041-1135 
Zeng, F. \& Yao, J. (2006). Ultrawideband impulse radio signal generation using a high-speed electrooptic phase modulator and a fiber-Bragg-grating-based frequency discriminator. IEEE Photonics Technology Letters, Vol. 18, No. 19, (October 2006) 2062-2064, ISSN 1041-1135

Zeng, F.; Wang, O. \& Yao, J.P. (2007). All-optical UWB impulse generation based on crossphase modulation and frequency discrimination. Electronic Letters, Vol. 43, No. 2, (January 2007) 119-121, ISSN 0013-5194 


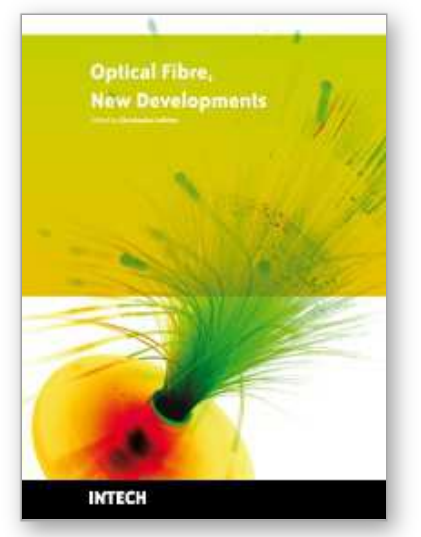

\section{Optical Fiber New Developments}

Edited by Christophe Lethien

ISBN 978-953-7619-50-3

Hard cover, 586 pages

Publisher InTech

Published online 01, December, 2009

Published in print edition December, 2009

The optical fibre technology is one of the hop topics developed in the beginning of the 21th century and could substantially benefit applications dealing with lighting, sensing and communication systems. Many improvements have been made in the past years to reduce the fibre attenuation and to improve the fibre performance. Nowadays, new applications have been developed over the scientific community and this book fits this paradigm. It summarizes the current status of know-how in optical fibre applications and represents a further source of information dealing with two main topics: the development of fibre optics sensors, and the application of optical fibre for telecommunication systems.

\section{How to reference}

In order to correctly reference this scholarly work, feel free to copy and paste the following:

Y. Ben Ezra, B.I. Lembrikov, M. Ran and M. Haridim (2009). All Optical Generation and Processing of IR UWB Signals, Optical Fiber New Developments, Christophe Lethien (Ed.), ISBN: 978-953-7619-50-3, InTech, Available from: http://www.intechopen.com/books/optical-fiber-new-developments/all-optical-generation-andprocessing-of-ir-uwb-signals

\section{INTECH}

open science | open minds

\section{InTech Europe}

University Campus STeP Ri

Slavka Krautzeka 83/A

51000 Rijeka, Croatia

Phone: +385 (51) 770447

Fax: +385 (51) 686166

www.intechopen.com

\section{InTech China}

Unit 405, Office Block, Hotel Equatorial Shanghai

No.65, Yan An Road (West), Shanghai, 200040, China

中国上海市延安西路65号上海国际贵都大饭店办公楼 405 单元

Phone: +86-21-62489820

Fax: $+86-21-62489821$ 
(C) 2009 The Author(s). Licensee IntechOpen. This chapter is distributed under the terms of the Creative Commons Attribution-NonCommercial-ShareAlike-3.0 License, which permits use, distribution and reproduction for non-commercial purposes, provided the original is properly cited and derivative works building on this content are distributed under the same license. 